\title{
Five days of head-down-tilt bed rest induces noninflammatory shedding of L-selectin
}

\author{
M. Feuerecker, ${ }^{1}$ B. Feuerecker, ${ }^{1}$ S. Matzel, ${ }^{1}$ M. Long, ${ }^{2}$ C. Strewe, ${ }^{1}$ I. Kaufmann, ${ }^{1}$ M. Hoerl, ${ }^{1}$ G. Schelling, ${ }^{1}$ \\ M. Rehm, ${ }^{1}$ and A. Choukèr ${ }^{1}$ \\ ${ }^{1}$ Department of Anaesthesiology, Klinikum Großhadern, University of Munich, Munich, Germany; and ${ }^{2}$ National Microgravity \\ Laboratory and Center for Biomechanics and Bioengineering, Institute of Mechanics, Chinese Academy of Sciences, \\ Beijing, China
}

Submitted 27 March 2013; accepted in final form 10 May 2013

Feuerecker M, Feuerecker B, Matzel S, Long M, Strewe C, Kaufmann I, Hoerl M, Schelling G, Rehm M, Choukèr A. Five days of head-down-tilt bed rest induces noninflammatory shedding of L-selectin. J Appl Physiol 115: 235-242, 2013. First published May 16, 2013; doi:10.1152/japplphysiol.00381.2013.-Head-down-tilt bed rest (HDTBR) is a popular model, simulating alterations of gravitation during space missions. The aim of this study was to obtain a better insight into the complexly orchestrated regulations of HDTBR-induced immunological responses, hypothesizing that artificial gravity can mitigate these HDTBR-related physiological effects. This crossover-designed 5 days of HDTBR study included three protocols with no, or daily $30 \mathrm{~min}$ of centrifugation or $6 \times 5$ min of centrifugation. Twelve healthy, male participants donated blood pre-HDTBR, post-HDTBR, and twice during HDTBR. Cellular immune changes were assessed either by enumerative and immune cell phenotyping assays or by functional testing of responses to either recall antigens or receptor-dependent activation by chemotactic agents $N$-formyl-methionyl-leucyl-phenylalanine (fMLP) and with TNF- $\alpha$. The expression of the adhesion molecule L-selectin (CD62L) on the surface of granulocytes and its shedding into plasma samples were measured. In parallel, other humoral factor, such as interleukin-6 and interleukin-8, parameters of endothelial damage (glycocalyx) were determined. Hematocrit and hemoglobin were significantly increased during HDTBR. Although immune functional tests did not indicate a change in the immune performance, the expression of CD62L on resting granulocytes was significantly shed by $50 \%$ during HDTBR. Although the latter is normally associated to an activation of inflammatory innate immune responses and during interaction of granulocytes with the endothelium, CD62L shedding was, however, not related either to a systemic inflammatory alteration or to shedding of the endothelial glycocalyx during bed rest. This suggests a noninflammatory or "mechanical" shedding related to fluid shifts during head-down intervention and not to an acute inflammatory process.

CD62L; shedding; immunological changes; short-term bed rest

IN THE COURSE OF SPACEFLIGHTS, the human body is facing many physiological and psychological challenges. To gain more insights in underlying mechanisms, many bed rest studies have been conducted on Earth in the last two decades as they are suspected to best mirror long-term microgravity on Earth. In line with these studies, head-down-tilt $\left(-6^{\circ}\right)$ bed rest could prove its reliability as a good simulation model for many physiological effects of spaceflight (28).

The numerous bed rest studies show quite consistent data referring to bone demineralization as well as to muscle loss (28). Also, cardiovascular changes as an effect of a redistribu-

Address for reprint requests and other correspondence: A. Choukèr, Dept. of Anaesthesiology, Univ. of Munich, Marchioninistrasse 15, 81377 Munich, Germany (e-mail: alexander.chouker@med.uni-muenchen.de). tion of body fluids are observed during bed rest (40). Nevertheless, a crucial factor, which is also one of the important concerns in human spaceflights, is the alteration of the immune system. The extreme environment itself or the cosmic radiation during space missions are factors influencing and weakening immunity $(32,35)$. Alhtough not all factors can be simulated on Earth, data on immune functions observed during headdown-tilt bed rest studies (HDTBR) are showing hereby only some of the effects of space and spaceflight conditions on the complexly orchestrated immune response. It was found in some bed rest studies that, aside from altered leukocyte subsets or changes in plasma cytokine levels, cellular immunity also was affected to different degrees $(5,11,31,38)$.

To obtain a better understanding in the acute regulations of immunological responses, the here-presented crossover-designed short-term bed rest study was performed. In addition, two of the three arms aimed to investigate the effects of artificial gravity in individuals exposed to bed rest, hypothesizing to provoke, through artificial gravity, a reversed effect of bed rest-induced physiological alterations.

\section{MATERIALS AND METHODS}

\section{Study design}

Study approval was obtained by the national ethics committee. All study participants signed a written, informed consent. In total, 12 healthy men were included in this crossover-designed investigation (Fig. 1; Table 1). The study was conducted at the Institute for Space Physiology and Medicine (MEDES) facility located in Toulouse, France. Participants were not allowed to eat or drink anything at least $60 \mathrm{~min}$ before sample collection. All protocols included, after a confined adaptation phase of 5 days [baseline data collection (BDC)], the bed rest at $-6^{\circ}$ head-down-tilt (HDTBR) for 5 days, followed by a "rehab" phase with no bed rest lasting 5 days (rehab/return $=\mathrm{R}$ ). Protocol 1 (control) had no intervention during HDTBR, whereas protocol 2 (artificial gravity $1=$ AG1) contained on each day of HDTBR 30 min of continuous centrifugation at $1 \mathrm{G}$ in the center of mass. Protocol 3 (AG2) differed from protocol 2 in that the centrifugation was performed six times for 5 min each with a short break in between (total time of $\sim 45 \mathrm{~min}$ ).

\section{General data}

These data include demographic data on the participants as well as information on the study performance.

\section{Immune monitoring}

Leukocyte differentiation. White blood cell count, granulocyte count, as well as lymphocyte/monocyte count were performed on EDTA-anticoagulated blood samples using a Coulter STKS (Coulter Electronics, Luton, UK). 


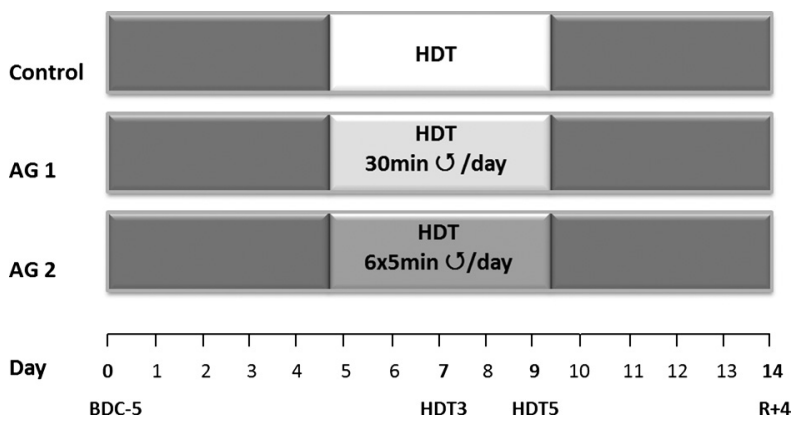

Fig. 1. Study protocol of the 5 days of short-term bed rest campaign (STBR). HDT, head-down tilt $\left(-6^{\circ}\right)$; $\mathcal{U}$, centrifugation at $1 \mathrm{G}$ in the center of mass; $\mathrm{BDC}$, baseline data collection; R, rehabilitation; AG, artificial gravity. Boldmarked days indicate measuring time points.

Further leukocyte differentiation was done using the BD Simultest, IMK kit lymphocyte (anti-CD3/CD4/CD8/CD16,56) (Becton Dickinson, Heidelberg, Germany). After antibody incubation and erythrocytal lysis, flow cytometric analysis of samples was performed using a Partec Cyflow SL (Partec, Münster, Germany).

Granulocytic activation marker. The adhesion molecule CD62L was measured on granulocytes using stained antibodies against this cluster of differentiation. The antibody was purchased from Becton Dickinson. Activity determination was done by using the flow cytometer Partec Cyflow SL.

In addition, soluble CD62L in plasma samples was determined using an ELISA kit (sL-selectin ELISA, IBL International, Hamburg, Germany).

\section{Hydrogen peroxide production of granulocytes}

$T N F-\alpha-/ f M L P$-induced production of hydrogen peroxide production of granulocytes. Leukocyte-enriched plasma containing $\sim 1 \times$ $10^{5}$ leukocytes was incubated with $1 \mu \mathrm{M}$ dihydrorhodamine 123 (DHR123; MoBiTec, Göttingen, Germany) in $0.5 \mathrm{ml}$ of HBSS (Pharmacy, University of Munich, Germany). After a 5-min priming of cells with TNF- $\alpha$, PMNLs were additionally stimulated by the application of $N$-formyl-methionyl-leucyl-phenylalanine (fMLP; SigmaAldrich) $\left(10^{-7} \mathrm{M}\right)$. Fifteen minutes after stimulatory initiation, activation of cells was stopped by placing the tubes in ice water. Production of $\mathrm{H}_{2} \mathrm{O}_{2}$ was measured using flow cytometry (Partec Cyflow SL).

Hydrogen peroxide plasma levels. The overall oxidative status was measured in plasma samples by a photometric test system according to the manufacturer's manual [PerOx (TOS/TOC) Kit, Immundiagnostik, Bensheim, Germany].

Plasma cytokines. Concentrations of EDTA plasma cytokines interleukin (IL)-6 (IL-6) and IL-8 were analyzed by Luminex xMAP technology (Bioplex) with commercially available reagents from Bio-Rad Laboratories, according to the manufacturer's guidelines. The data were analyzed using Bioplex Software, in which sensitivity is $1.9 \mathrm{pg} / \mathrm{ml}$ for the analyzed cytokines.

Cytokine release assay. Whole lithium-heparinized blood $(500 \mu \mathrm{l})$ was transferred under aseptic conditions into tubes prefilled with an equal volume $(500 \mu \mathrm{l})$ of DMEM Nutrient Mixture F-12 HAM (Sigma-Aldrich) and the different stimulants $(1,000-\mu l$ total assay volume). The assay tubes contained DMEM and either a bacteria antigen mixture containing diphterie-, tetanus-, and pertussis-toxoid (all three combined in 1\% Boostrix, GlaxoSmithKline, Munich, Germany) or viral antigen mixture containing cytomegalovirus (CMV)lysate $(10 \mu \mathrm{g} / \mathrm{ml}$; ABI, Columbia, OH), Epstein-Barr virus (EBV)lysate $(10 \mu \mathrm{g} / \mathrm{ml}$; ABI), influenza proteins (1\% Influvac, Solvay, Hannover, Germany), or pokeweed mitogen (PWM; $5 \mu \mathrm{g} / \mathrm{ml}$; SigmaAldrich) as positive control.
The assay tubes were incubated altogether for $48 \mathrm{~h}$ at $37^{\circ} \mathrm{C}$. Supernatant plasma was transferred into Eppendorf tubes and immediately frozen at $-80^{\circ} \mathrm{C}$ for future cytokine analyses (see Cytokines). The readout cytokine was IL-2.

\section{Fluid homeostasis}

Cellular blood parameters. Hematocrit, hemoglobin, and mean corpuscular volume (MCV) were determined on EDTA-anticoagulated blood samples using a Coulter STKS (Coulter Electronics, Luton, UK).

Fluid characteristics. Fluid consumption and urine production were monitored on the respective time points [HDTBR before BDC (BDC-5), day 3 of HDT (HDT3), day 5 of HDT (HDT5), and day 3 of rehab $(\mathrm{R}+3)]$. The hereby collected data were used for the calculation of the daily fluid balance.

Determination of endothelial glycocalyx components. Heparan sulfate and hyaluronic acid, two key structural components of the endothelial glycocalyx (30), were measured in plasma samples at the respective time points $(n=4)$. ELISA kits were used to analyze these two components according to the manufacturers' manuals (Seikagaku, Tokyo, Japan for heparan sulfate and Echelon Biosciences, Salt Lake City, UT for hyaluronic acid).

\section{Statistical analysis}

Data were analyzed with the commercially available software SPSS 20.0 (IBM, New York) and Sigma Plot 11.0 (Chicago, IL). Normal distribution was tested by the Kolmogorov-Smirnov test. Changes in parameters across the four time points of measurement were analyzed with a repeated-measurement general linear model (RM-GLM) with time point as a within-subject variable and group assignment as a between-subject variable. Fisher's least significant difference (LSD) post hoc test was used to determine which groups differed significantly from each other. Additionally, within-group analyses across the four time points were performed using one-way RM ANOVA followed by a Fisher's LSD post hoc test. Demographic variables between groups were compared by ANOVA. Data are considered to be significantly different at $P<0.05$. Results are expressed as means $\pm \mathrm{SD}$, except data presented in figures, where means $\pm \mathrm{SE}$ are drawn for a clearer display.

\section{RESULTS}

\section{General data}

All volunteers tolerated the HDTBR period well. Aside from one incident during the centrifugation procedure, in which the centrifugation had to be stopped after $17 \mathrm{~min}$ due to the development of extreme bradycardia and almost the loss of consciousness, no major centrifugation-related incidents were reported. Due to an acute appendicitis, one participant had to be excluded from the respective study arm. After recovery, he

Table 1. Demographical data

\begin{tabular}{lccc}
\hline \hline \multicolumn{1}{c}{ Participants } & $\begin{array}{c}\text { Protocol } 1 \\
(\text { Control; } n=13)\end{array}$ & $\begin{array}{c}\text { Protocol 2 } \\
(\mathrm{AG} 1 ; n=12)\end{array}$ & $\begin{array}{c}\text { Protocol 3 } \\
(\mathrm{AG} 2 ; n=11)\end{array}$ \\
\hline Age, yr & $31.69 \pm 7.55$ & $32.58 \pm 7.14$ & $33.64 \pm 6.44$ \\
Size, m & $1.77 \pm 0.081$ & $1.78 \pm 0.077$ & $1.79 \pm 0.072$ \\
Weight, kg & $73.92 \pm 8.41$ & $75.08 \pm 7.60$ & $76.46 \pm 6.20$ \\
Body mass index & $23.82 \pm 1.74$ & $23.99 \pm 1.69$ & $24.20 \pm 1.60$ \\
\hline
\end{tabular}

Data are means \pm SD. Control group, head-down-tilt bed rest (HDTBR) without artificial gravity; AG1 group, HDTBR with $30 \mathrm{~min}$ of continuous artificial gravity (AG) on each HDTBR day; AG2 group, HDTBR with six 5 -min periods of discontinuous AG on each HDTBR day. No significant differences were observed. 
Table 2. Leukocyte differentiation

\begin{tabular}{|c|c|c|c|c|}
\hline & Time Points & Protocol 1 (Control) & Protocol 2 (AG1) & Protocol 3 (AG2) \\
\hline \multirow{2}{*}{ Leukocytes, G/1 } & HDT3 & $5.96 \pm 1.40$ & $6.12 \pm 0.94$ & $6.02 \pm 0.93$ \\
\hline & HDT5 & $5.94 \pm 1.10$ & $6.06 \pm 1.24$ & $6.05 \pm 0.90$ \\
\hline \multirow{3}{*}{ PMN, G/1 } & BDC-5 & $3.23 \pm 0.95$ & $3.37 \pm 1.21$ & $3.04 \pm 0.76$ \\
\hline & HDT3 & $3.59 \pm 1.11$ & $3.71 \pm 0.86$ & $3.58 \pm 0.80 \dagger$ \\
\hline & HDT5 & $3.50 \pm 0.99$ & $3.62 \pm 1.13$ & $3.41 \pm 0.78 \dagger$ \\
\hline \multirow{3}{*}{ Monocytes, G/l } & HDT3 & $0.48 \pm 0.14$ & $0.50 \pm 0.12$ & $0.50 \pm 0.10$ \\
\hline & HDT5 & $0.49 \pm 0.13$ & $0.46 \pm 0.11$ & $0.49 \pm 0.11$ \\
\hline & $\mathrm{R}+4$ & $0.48 \pm 0.14$ & $0.45 \pm 0.11$ & $0.45 \pm 0.13$ \\
\hline \multirow{4}{*}{ B-lymphocytes, \% } & BDC-5 & $10.28 \pm 2.60$ & $10.68 \pm 3.85$ & $10.24 \pm 4.10$ \\
\hline & HDT3 & $9.41 \pm 4.67$ & $10.10 \pm 2.59$ & $9.67 \pm 4.44$ \\
\hline & HDT5 & $9.88 \pm 3.68$ & $10.26 \pm 3.52$ & $10.14 \pm 4.03$ \\
\hline & $\mathrm{R}+4$ & $11.00 \pm 4.26$ & $10.75 \pm 3.59$ & $11.47 \pm 4.40$ \\
\hline \multirow{3}{*}{$\mathrm{CD} 3+/ \mathrm{CD} 4+$} & HDT3 & $37.24 \pm 13.70$ & $40.10 \pm 12.34$ & $39.06 \pm 7.92$ \\
\hline & HDT5 & $37.32 \pm 11.93$ & $39.48 \pm 12.25$ & $38.87 \pm 9.95$ \\
\hline & $\mathrm{R}+4$ & $41.80 \pm 14.72$ & $41.88 \pm 10.81$ & $38.58 \pm 10.62$ \\
\hline \multirow[t]{4}{*}{$\mathrm{CD} 3+/ \mathrm{CD} 8+$} & BDC-5 & $14.37 \pm 5.14$ & $16.85 \pm 8.45$ & $12.55 \pm 2.84$ \\
\hline & HDT3 & $22.01 \pm 15.58$ & $23.62 \pm 14.07 *$ & $17.96 \pm 8.27$ \\
\hline & HDT5 & $23.17 \pm 11.00$ & $23.00 \pm 11.84^{*}$ & $18.65 \pm 10.78$ \\
\hline & $\mathrm{R}+4$ & $24.56 \pm 17.26$ & $25.64 \pm 16.08 *$ & $17.85 \pm 10.01$ \\
\hline \multirow[t]{4}{*}{$\mathrm{CD} 3-/ \mathrm{CD} 16+56+$} & BDC-5 & $10.48 \pm 3.03$ & $9.81 \pm 4.21$ & $9.40 \pm 3.48$ \\
\hline & HDT3 & $11.72 \pm 5.85$ & $11.25 \pm 4.54$ & $11.10 \pm 4.37$ \\
\hline & HDT5 & $12.06 \pm 5.94$ & $12.24 \pm 4.09$ & $11.72 \pm 3.70$ \\
\hline & $\mathrm{R}+4$ & $10.89 \pm 4.67$ & $10.12 \pm 2.69$ & $10.53 \pm 3.80$ \\
\hline \multirow[t]{2}{*}{$\mathrm{CD} 3+/ \mathrm{CD} 16+56+$} & BDC-5 & $10.91 \pm 7.57$ & $9.20 \pm 8.98$ & $8.33 \pm 7.95$ \\
\hline & HDT3 & $16.34 \pm 15.96$ & $12.29 \pm 12.55$ & $8.32 \pm 8.64$ \\
\hline
\end{tabular}

Data are means \pm SD. BDC-5, HDTBR before baseline data collection (BDC); HDT3, day 3 of HDTBR; HDT5, day 5 of HDTBR; R + 3, day 3 of rehab. *Significant difference vs. BDC-5 in the respective group $(P<0.05)$. $†$ Significant difference vs. BDC-5 and $\mathrm{R}+4(P<0.05)$. There were no statistically significant differences between different study groups.

was included several weeks later in the last tier of the study. Because of the crossover design, this subject could only be re-included into protocol 1 (control). For this reason, the groups consist of different group sizes $(n=11-13)$ (Table 1$)$. The variable group sizes had no influence on the statistical outcome.

The applied dietetic regimen did not differ between the study protocols (data not shown).

\section{Immune monitoring}

Leukocyte differentiation. Leukocyte counts remained within a small normal point-to-point variability during observation period. The polymorphonuclear leukocytes (PMN) showed a slight increase at HDTBR days, which reached the level of statistical significance in the discontinuous centrifugation protocol. The percentage of B-lymphocytes did not show major alterations. T-lymphocyte percentages decreased over time, reaching the level of statistical significance in the control group. T-lymphocyte subpopulations showed an increase compared with the BDC most prominently in the CD8+ subpopulation, followed by CD4+ and CD16+56+ cells. In addition, CD3-/CD16+56+ cells were augmented during HDTBR and peaked at HDT5 (Table 2).
Granulocytic activation marker. CD62L on granulocytes showed in all protocols at HDT3 a significant decrease. This effect was entirely reversed at HDT5 and R +4 , and BDC values were reached (Fig. 2).

In correlation to the CD62L shedding on granulocytes, soluble CD62L proteins were increased on HDT3 and HDT5 in

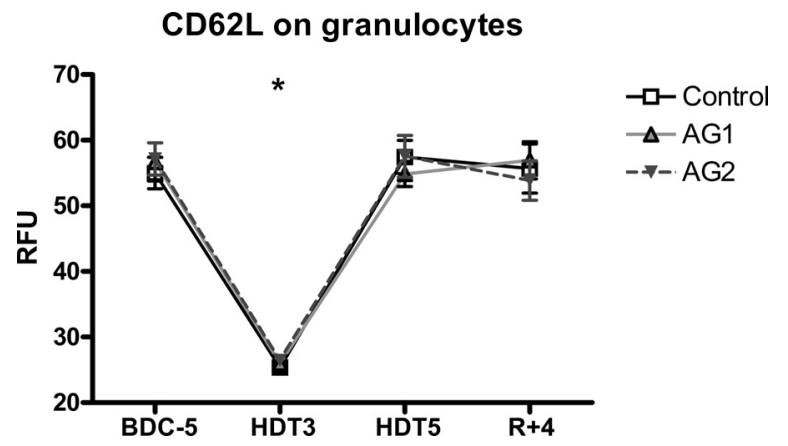

Fig. 2. Activation marker (CD62L on granulocytes). Data are means $\pm \mathrm{SE}$. RFU, relative fluorescence units; BDC-5, baseline data collection 5 days before HDT; HDT3, day 3 of HDT; HDT5, day 5 of HDT; R + 4, day 4 of rehab. *Significant difference in all study groups vs. BDC-5, HDT 5 , and R+ $4(P<0.05)$. There were no statistical differences between the study groups. 
Table 3. Activation marker (soluble plasma CD62L level), hydrogen peroxide plasma levels, and plasma cytokines IL-6 and $I L-8$

\begin{tabular}{lcccc}
\hline \hline & Time Points & Protocol 1 (Control) & Protocol 2 (AG1) & Protocol 3 (AG2) \\
\hline Soluble CD62L, ng/ml & BDC-5 & $3,355.05 \pm 620.72$ & $3,498.13 \pm 521.89$ & $3,589.22 \pm 627.82$ \\
& HDT3 & $\mathbf{3 , 8 6 9 . 1 7} \pm \mathbf{5 0 6 . 4 2} *$ & $\mathbf{3 , 8 7 2 . 0 0} \pm \mathbf{6 9 4 . 6 4} *$ & $3,936.66 \pm 509.49(P=0.077)$ \\
& HDT5 & $\mathbf{3 , 7 2 8 . 6 3} \pm \mathbf{4 7 6 . 2 1} *$ & $\mathbf{3 , 9 4 0 . 3 7} \pm \mathbf{6 2 1 . 2 4} \boldsymbol{*}^{*}$ & $3,789.26 \pm 505.06$ \\
Perox, $\mu \mathrm{M}$ & $\mathrm{R}+4$ & $\mathbf{3 , 8 4 7 . 6 0} \pm \mathbf{7 2 2 . 1 4 *}$ & $3,592.53 \pm 757.83$ & $3,511.80 \pm 656.74$ \\
& BDC-5 & $409.75 \pm 226.02$ & $476.64 \pm 186.28$ & $358.55 \pm 125.08$ \\
& HDT3 & $461.18 \pm 206.57$ & $427.28 \pm 229.04$ & $346.51 \pm 128.98$ \\
IL-6, pg/ml & HDT5 & $427.35 \pm 212.62$ & $446.30 \pm 209.07$ & $397.94 \pm 145.19$ \\
& R +4 & $408.13 \pm 180.21$ & $418.59 \pm 216.31$ & $370.90 \pm 127.25$ \\
& BDC-5 & $1.24 \pm 0.76$ & $1.77 \pm 0.82$ & $1.04 \pm 0.64$ \\
IL-8, pg/ml & HDT3 & $1.22 \pm 0.47$ & $1.23 \pm 0.59$ & $1.10 \pm 0.66$ \\
& HDT5 & $1.18 \pm 0.44$ & $1.11 \pm 0.76$ & $1.33 \pm 0.41$ \\
& R +4 & $0.95 \pm 0.34$ & $2.72 \pm 0.58$ & $0.95 \pm 0.35$ \\
& BDC-5 & $2.29 \pm 0.64$ & $2.33 \pm 1.03$ & $2.30 \pm 1.00$ \\
& HDT3 & $2.03 \pm 0.67$ & $1.80 \pm 0.79$ & $2.28 \pm 1.22$ \\
& HDT5 & $2.14 \pm 0.62$ & $2.32 \pm 1.11$ & $2.26 \pm 0.71$ \\
\end{tabular}

Data are means \pm SD. *Significant difference vs. BDC-5 (differences in the respective group are marked in bold) $(P<0.05)$. $†$ Significant difference vs. $R+4(P<0.05)$. No statistically significant differences between the study groups were detectable.

all groups (Table 3). The level of statistical significance was reached in the control group and the continuous centrifugation group (AG1).

Hydrogen peroxide production of granulocytes. PMNs do have a tissue toxic capability/effect by producing $\mathrm{H}_{2} \mathrm{O}_{2}$ upon activation. This effect was provoked by two receptor-dependent stimuli: fMLP in combination with TNF- $\alpha$. Stimulation with fMLP and TNF- $\alpha$ led to a significant increase in $\mathrm{H}_{2} \mathrm{O}_{2}$ production. In the course of the study, this pattern did not change significantly. Also, the different protocols showed a similar trend and did not differ from each other [all groups: spontaneous $\mathrm{H}_{2} \mathrm{O}_{2}$-production values of $82.97 \pm 45.99$ (BDC-5), $105.53 \pm 59.02$ (HDT3), $98.08 \pm 39.15$ (HDT5), and $102.10 \pm 47.80(\mathrm{R}+4)$; TNF- $\alpha /$ fMLP stimulation values of $501.56 \pm 104.63$ (BDC-5), $511.33 \pm 128.90$ (HDT3), $525.64 \pm 106.23$ (HDT5), and 532.06 $\pm 107.97(\mathrm{R}+4)]$.

Hydrogen peroxide plasma levels. Hydrogen peroxide plasma levels did not change significantly during HDTBR (Table 3).

Plasma cytokines. Plasma IL- 6 and IL-8 showed no significant changes either over time or between study groups (Table 3 ).

Cytokine release assay. IL-2 response after stimulation with either bacterial or viral antigen stimulation in diluted whole blood remained unaffected after bed rest. This was similar in all study groups. Also, the maximal stimulation with pokeweed mitogen did not differ after the bed-rest period (Table 4).

\section{Fluid homeostasis}

Cellular blood parameters. During HDTBR, hemoglobin and hematocrit significantly increased irrespective to the dif- ferent study groups. On $\mathrm{R}+4$, previous values were achieved. The mean corpuscular volume did not change during the observation period (Table 5).

Fluid characteristics. During HDTBR, a significant reduction of fluid consumption was detectable irrespective to the study group. The lower water intake, compared with baseline, was persistent throughout the entire head-down-tilt period. Values returned to baseline after the end of bed rest at $\mathrm{R}+3$. According to the lower fluid intake, the diuresis was also reduced during the HDTBR period. Overall, the fluid balance was slightly but not significantly lowered during the investigation period (Fig. 3). The study groups did not significantly differ from each other.

Endothelial glycocalyx components. The endothelial glycocalyx components heparan sulfate and hyaluronate showed no changes in relation to HDTBR itself or to head-down-tilt and intervention days (Table 6).

\section{DISCUSSION}

The present study was designed to get a better insight to the immunological effects during short-term HDTBR as a model for spaceflights. Additionally, two different artificial gravity protocols were applied in this crossover-designed study to determine possible neutralizing or beneficial effects on immunological parameters (Fig. 1).

From all examined innate and adaptive immune parameters, the most interesting finding was that short-term bed rest led to a significant shedding of L-selectin (CD62L) on granulocytes at the initial phase of the HDTBR phase. The observed shed-

Table 4. Cytokine release assay

\begin{tabular}{llcrrr}
\hline \hline \multicolumn{1}{c}{ Recall Antigens } & Cytokine & Time Points & Protocol 1 (Control) & Protocol 2 (AG1) & Protocol 3 (AG2) \\
\hline Bacteria mix & IL-2, pg/ml & BDC-5 & $88.81 \pm 94.71$ & $96.23 \pm 154.70$ & $95.09 \pm 87.28$ \\
& & $\mathrm{R}+4$ & $85.74 \pm 123.14$ & $117.83 \pm 238.36$ & $89.55 \pm 108.37$ \\
Virus mix & $\mathrm{IL}-2, \mathrm{pg} / \mathrm{ml}$ & $\mathrm{BDC}-5$ & $271.48 \pm 365.86$ & $176.34 \pm 166.48$ & $287.10 \pm 301.79$ \\
& & $\mathrm{R}+4$ & $246.70 \pm 313.99$ & $222.47 \pm 242.86$ & $258.95 \pm 328.84$ \\
Pokeweed mitogen & $\mathrm{IL}-2, \mathrm{pg} / \mathrm{ml}$ & $\mathrm{BDC}-5$ & $1,691.81 \pm 1,602.64$ & $1,347.85 \pm 1,459.12$ & $1,043.49 \pm 508.52$ \\
& & $\mathrm{R}+4$ & $1,517.62 \pm 894.82$ & $925.87 \pm 436.08$ \\
\hline
\end{tabular}

Data are means \pm SD. IL-2 release was not significantly influenced during the observation period. 
Table 5. Cellular blood parameters

\begin{tabular}{|c|c|c|c|c|}
\hline & $\begin{array}{l}\text { Time } \\
\text { Points }\end{array}$ & $\begin{array}{l}\text { Protocol } 1 \\
\text { (Control) }\end{array}$ & $\begin{array}{l}\text { Protocol } 2 \\
\text { (AG1) }\end{array}$ & $\begin{array}{c}\text { Protocol } 3 \\
\text { (AG2) }\end{array}$ \\
\hline \multirow{4}{*}{$\begin{array}{l}\text { Hemoglobin, } \\
\mathrm{g} / \mathrm{dl}\end{array}$} & BDC-5 & $14.54 \pm 0.81$ & $14.69 \pm 1.03$ & $14.63 \pm 0.55$ \\
\hline & HDT3 & $15.78 \pm 0.84^{*}$ & $15.79 \pm 1.18 *$ & $16.20 \pm 0.88^{*}$ \\
\hline & HDT5 & $16.00 \pm 0.97 *$ & $16.09 \pm 1.31 *$ & $16.11 \pm 0.97 *$ \\
\hline & $R+4$ & $14.86 \pm 0.71$ & $14.53 \pm 0.93$ & $14.49 \pm 0.74$ \\
\hline \multirow[t]{4}{*}{ Hematocrit, \% } & BDC-5 & $43.22 \pm 1.63$ & $43.41 \pm 2.87$ & $42.67 \pm 1.68$ \\
\hline & HDT3 & $46.37 \pm 3.33^{*}$ & $47.40 \pm 3.90 *$ & $48.15 \pm 3.38 *$ \\
\hline & HDT5 & $46.53 \pm 2.96^{*}$ & $46.73 \pm 4.37 *$ & $46.34 \pm 2.85^{*}$ \\
\hline & $\mathrm{R}+4$ & $43.34 \pm 2.29$ & $42.68 \pm 2.57$ & $42.34 \pm 2.21$ \\
\hline \multirow[t]{4}{*}{$\mathrm{MCV}, \mathrm{fl}$} & BDC-5 & $86.23 \pm 3.68$ & $86.50 \pm 2.81$ & $86.09 \pm 3.39$ \\
\hline & HDT3 & $86.50 \pm 4.40$ & $87.92 \pm 5.05$ & $88.18 \pm 4.58$ \\
\hline & HDT5 & $85.50 \pm 3.06$ & $85.17 \pm 2.59$ & $85.27 \pm 3.17$ \\
\hline & $R+4$ & $85.67 \pm 3.42$ & $85.92 \pm 3.18$ & $86.36 \pm 3.56$ \\
\hline
\end{tabular}

Data are means $\pm \mathrm{SD}$. MCV, mean corpuscular volume. *Significant difference vs. BDC-5 and $R+4(P<0.05)$. There were no statistical significant differences between the groups. Significant differences within groups are marked in bold.

ding on granulocytes was consistent with the findings in plasma samples as the soluble CD62L molecules were hereby increased. All protocols showed the same effect on CD62L. Artificial gravity protocols were not able to neutralize this alteration.

\section{Shedding of CD62L as a consequence of inflammation or immune dysfunction?}

L-selectin, or CD62L, plays a substantial role during inflammatory processes in leukocytic adherence to vascular endothelial cells as well as to platelets $(17,34,36)$. To avoid an overshooting leukocyte response, the expression and the function of L-selectin are well controlled and occur only with directly surrounding tissue (17). In the light of the actual finding and the general functions of this adhesion molecule CD62L, the question is now: Why has this shedding occurred?

L-selectin is normally shed under diverse pathophysiological states, especially under inflammatory conditions. Shedding of CD62L can be seen, e.g., in patients suffering from fibromyalgia, leading to a potentially compromised host defense (20). Another situation in which a decrease of this adhesion molecule can be detected is in critically ill patients suffering from systemic inflammation $(1,19,26)$. With the broad panel of immunological parameters of innate and adaptive immunity as analyzed during HDTBR, no indication of an inflammatory process was detectable. A global, although unspecific, marker for inflammation is the overall leukocyte count (29). This parameter remained stable during the entire study, and also no alterations could be seen in the monocyte count as well as in the percentage of B-lymphocytes, whereas PMNs marginally increased during HDTBR. Furthermore, the percentage of T-lymphocytes tended to decrease over time, including a population shift with slightly increased T-helper (CD4+/CD8-) and cytotoxic T-cells (CD4-/CD8+). All these variations are seen as a physiological variation in a normal healthy population. More sensitive early phase infection markers such as the plasma cytokines IL-6 and IL-8 (29) also remained unaffected at low baseline values at all time points. Since cell counts and cytokines have only a describing character and lack the ability to provide the state of the functionality of the immune system, we have investigated two central functional properties of the innate as well as the adaptive immune function. The first line of host defense is maintained by cells of the innate immune system and the capability of the polymorphonuclear leukocytes to produce hydrogen peroxide (24). This answer was tested during bed rest by the reactivity of PMNs to generate $\mathrm{H}_{2} \mathrm{O}_{2}$ upon receptor stimulation with fMLP and TNF- $\alpha$ and by the peroxide concentrations in plasma samples. Neither an increase in the oxidative reactivity nor a significant decreased reactivity of PMNs to stimulation could be quantified. In conclusion, polymorphonuclear leukocytes are not affected under shortterm bed-rest conditions in their capability to produce hydrogen peroxide. To display a key function of adaptive immunity, a cytokine release assay was applied, monitoring especially the
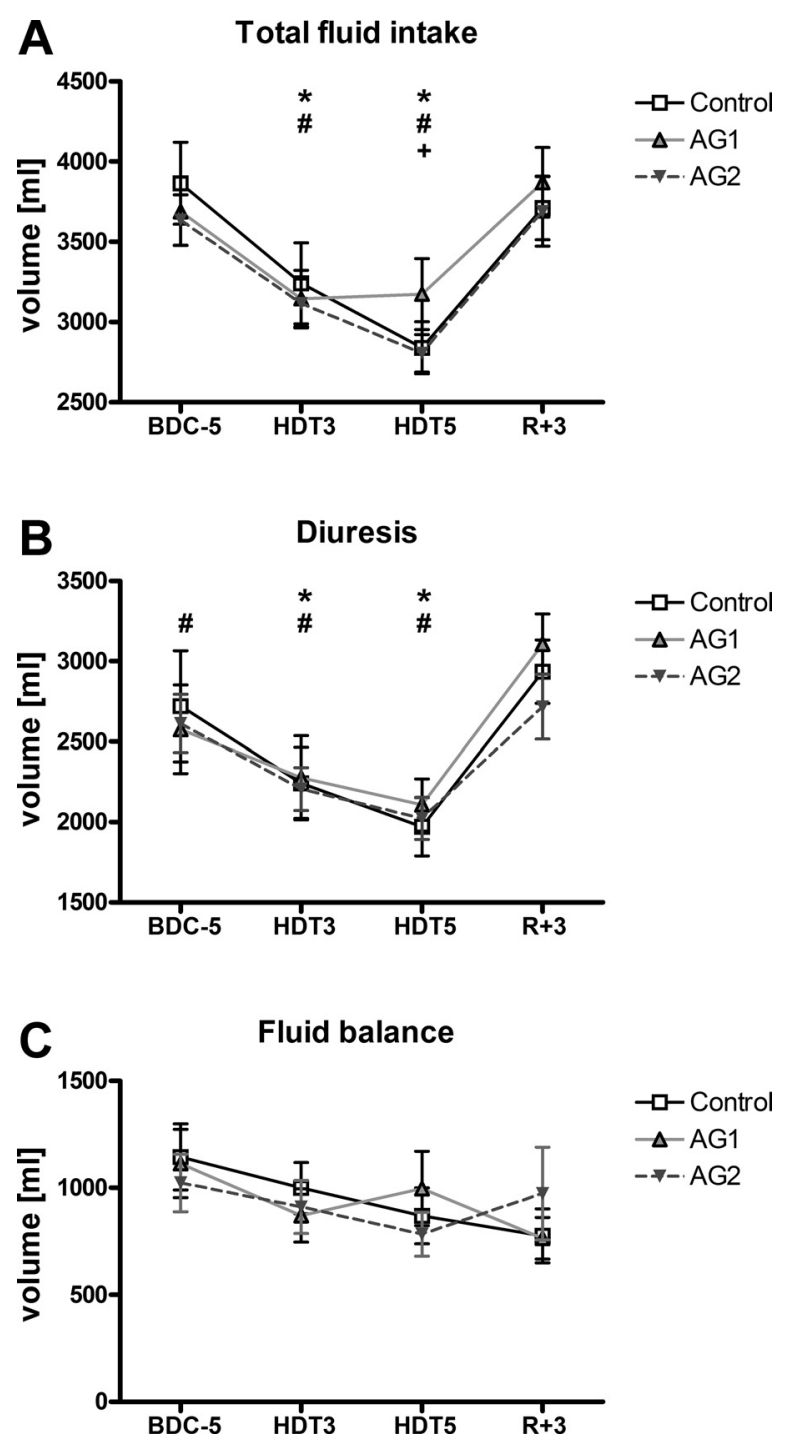

Fig. 3. Fluid characteristics during HDTBR at BDC-5, HDT3, HDT5, and R + 3. Data are means \pm SE. No significant differences were seen between the study protocols. A: total water intake. *Significant difference compared with BDC-5 (all groups) $(P<0.05)$. \#Significant difference compared with $\mathrm{R}+3$ (all groups) $(P<0.05)$. + Significant difference compared with HDT3 (control group) $(P<0.05)$. $B$ : diuresis. ${ }^{*}$ Significant difference compared with BDC-5 (control and AG2 group) $(P<0.05)$. \#Significant difference compared with $\mathrm{R}+3$ (all groups) $(P<0.05)$. $C$ : fluid balance. No significant changes within or between groups (general linear model; repeated-measures ANOVA with Fisher's least-significant difference post hoc test). 
Table 6. Components of the endothelial glycocalyx

\begin{tabular}{ccccc}
\hline \hline & $\begin{array}{c}\text { Time } \\
\text { Points }\end{array}$ & $\begin{array}{c}\text { Protocol 1 } \\
\text { (Control) }\end{array}$ & $\begin{array}{c}\text { Protocol 2 } \\
\text { (AG1) }\end{array}$ & $\begin{array}{c}\text { Protocol 3 } \\
\text { (AG2) }\end{array}$ \\
\hline Heparan & BDC-5 & $6.97 \pm 1.56$ & $7.99 \pm 1.79$ & $7.69 \pm 1.04$ \\
sulfate, & HDT3 & $8.34 \pm 2.85$ & $7.43 \pm 2.29$ & $7.23 \pm 1.27$ \\
$\mu \mathrm{g} / \mathrm{ml}$ & HDT5 & $7.74 \pm 2.73$ & $8.16 \pm 3.47$ & $7.26 \pm 1.71$ \\
& $\mathrm{R}+4$ & $6.84 \pm 0.88$ & $8.23 \pm 3.59$ & $7.41 \pm 0.92$ \\
Hyaluronate, & BDC-5 & $112.92 \pm 47.40$ & $99.60 \pm 39.96$ & $88.13 \pm 41.92$ \\
ng/ml & HDT3 & $95.86 \pm 50.49$ & $91.12 \pm 60.42$ & $76.85 \pm 28.51$ \\
& HDT5 & $86.70 \pm 9.40$ & $84.39 \pm 27.12$ & $84.84 \pm 27.41$ \\
& R +4 & $90.00 \pm 22.19$ & $108.34 \pm 28.06$ & $105.14 \pm 43.26$ \\
\hline
\end{tabular}

Data are means \pm SD. There are no significant differences within or between the study protocols.

T-cell-triggered cytokine response toward recall antigens (10, 18). After $48 \mathrm{~h}$ of incubation of whole blood with either a bacterial or a viral antigen mixture, IL-2 was assessed in the respective supernatant. Irrespective from the exposed antigen stimulation, cytokine production did not differ significantly after the end of bed rest from the baseline values. Another important trigger in inflammatory processes is tissue hypoxia, and Eltzschig et al. (9) recently summarized where and how hypoxia can induce inflammation. As in the HDTBR, no cytokine release or granulocytic hydrogen peroxide production was measurable, it can indirectly be assumed that no hypoxia or local hypoxic conditions were present. Of course, an ultimate proof of hypoxic tissue conditions can be given only either by monitoring tissue $\mathrm{Po}_{2}$ or from cellular analyses from tissue biopsies. However, the absolute absence of any inflammation or activation of the innate or adaptive immunity makes the hypoxic trigger rather unlikely.

Only few data are available on immunity under bed-rest conditions. To the best of our knowledge, no adequate data on immunological alterations are available for short-term bed-rest studies. Kelsen et al. have shown in a study of 21 days of lasting head-down bed-rest that the cytokine response to polyclonal T-cell stimulation is weakened (21). Since no altered cytokine response could be measured in the study presented here, it must be speculated that the HDTBR duration was too short to reproduce such findings. One explanation for the inconsistency and not entire comparability of the immune data gathered from previous HDTBR data might be due to different study setups with, e.g., long-term bed-rest periods or interventional procedures in between.

Taking these findings together, no major immune alterations, neither immune suppression nor immune activation, were observed during bed rest alone or as function of the artificial gravitation interventions, respectively.

\section{Shedding of CD62L as a consequence of "noninflammatory" changes?}

Because inflammation as a possible reason for the CD62L shedding does subsequently not apply here, other nonimmune mechanisms need to be considered responsible for the shedding of L-selectin.

Shedding of selectins can result as a function of inflammation but can also be provoked by mechanical shear stress, such as by fluid distribution changes $(4,22,23,25,27)$. To determine such a possible fluid redistribution, we have investigated on the one hand fluid volume profiles and on the other hand blood parameters like hemoglobin, hematocrit, and mean corpuscular volume, possibly reflecting hemo-concentration. Overall, the fluid balance was only slightly altered during HDTBR. Although participants consumed lower amounts of fluids, the diuresis was compensatorily diminished, resulting in an almost unaffected fluid balance. Our findings of a hemo-concentration are consistent with those from Drummer et al. (8), who have shown a fluid redistribution from the intra- and extravascular space. The described acute intravascular fluid loss can be indirectly seen here by the hemo-concentration. Hematocrit and hemoglobin significantly increased during the head-downtilt period, whereas the mean corpuscular volume as a measure of the average red blood cell size was unaffected. HDTBR studies investigating plasma volume variations with or without specific dyes $(6,16,33)$ revealed the commonality of a rapid descent of plasma volume up to $10 \%$ within the first days of HDTBR. Moreover, Johansen et al. (16) reported that the plasma volume can be reliably reflected by changes in the hematocrit and hemoglobin concentration. Transferring this correlation to the here-presented data, a significant reduction in plasma volume also occurred in this investigation. Using the commonly described formula \{change of plasma volume $(\%)=\left[\mathrm{Hb}_{\mathrm{BDC}} / \mathrm{Hb}_{\mathrm{HDT}} \times\left(100-\mathrm{Hct}_{\mathrm{HDT}}\right) /\left(100-\mathrm{Hct}_{\mathrm{BDC}}\right)-\right.$ $1] \times 100\}(7,15)$ and calculating the plasma volume alteration during the head-down-tilt period, displays an average plasma volume reduction of up to $15 \%$ on both HDT collection days. This finding suggests, according to the reports on shearinduced shedding of L-selectin $(22,27)$, that this phenomenon could be caused by the shear forces provoked by fluid redistribution. To confirm whether the sole fluid redistribution is responsible for the shedding of CD62L or whether this is due to changes of the integrity of the endothelial layer, we have additionally investigated components of the endothelial glycocalyx (EG). The EG is a thin layer on endothelial cells composed of different membrane-bound macromolecules such as sulfated proteoglycans or hyaluronate $(30,39)$. The EG plays a pivotal role in the modulation of the oncotic forces, the interaction with leukocytes and respective adhesion molecules, as well as in the initiation of inflammatory responses. Hereby, this fragile layer can be easily affected by volume redistribution in the human body. Exogenous applied volume infusions (3) as well as endogenous conditions for example ischemia/ reperfusion (2) conditions are able to severely damage the EG layer, leading to an enforced interaction with leukocytes, including adhesion molecules. Two components of the EG, which are easily and altered very early by endothelial surface interactions, are heparan sulfate and hyaluronate (39). For this reason, we have looked on those components as they are either destroyed by volume shifts (3) or affected by shear stress (37, 39). Interestingly, these two EG components, heparan sulfate and hyaluronate, were not altered during the entire examination. Since the plasma volume and the endothelial integrity are only two of multiple indicators for the alteration of physiological blood flow, it is hard to direct, from these hematological measurements, the conclusion that no important/major forces, either through mechanotransduction or fluid shear stress, have been existent. In fact, blood flow produces the different shear stresses (magnitude) and flow patterns (steady, pulsatile, or oscillatory) along the circulation. Meanwhile, it has been known that mechanical shedding occurs in an in vitro study (23). Thus it is most likely that the short-term bed rest does 
modify the blood flow via fluid redistribution and induces the transient L-selectin shedding. Hence, it is not an inflammatory but an adaptive stress response.

Therefore, it can only be speculated why a shedding of CD62L occurred. As described above, CD62L adhesion molecules are shed by either inflammatory processes or mechanical forces. Because inflammation could be excluded as a source of L-selectin shedding, fluid redistribution might have caused and contributed to the L-selectin shedding. This, however, was not associated with alterations of the fragile EG layer. It has to be confessed that shedding of L-selectin CD62L from granulocytes has to be seen as a noninflammatory shedding, most likely related and triggered by not exactly known local or systemic mechanical or other stimulatory factors. The pathophysiological impact remains unclear but might contribute to a higher tethering and rolling of polymorphonuclear leukocytes to the endothelial wall. This shedding might be a potential early alert signal of the human body to possibly upcoming inflammatory events. In the postcapillary venous low pressure system, different conditions, such as substrate exchange, larger pores, or smaller glycocalyx, can be found (13). It is possible that CD62L-shed granulocytes might be "stored" in this particular system as an emigration to the tissue is facilitated. If an inflammation will be missing, a further chemotaxis will not be initiated (12).

The rolling dynamics of activated PMNs is determined by two opposite impacts: reducing the surface presentation would enhance the rolling but lowering the kinetic rates would decrease the rolling (14). Such transient L-selectin shedding is presumably used to be responsible for the mechanical shear induced by fluid redistribution.

\section{Limitations}

Fluid shifts as discussed were only indirectly assessed in this investigation since no plasma volume determination with dyes was performed. Blood draws and therefore the immune tests could not be performed daily/at all time points for ethical reasons and blood volume restrictions, respectively.

\section{Conclusions}

Five days of short-term bed rest induces a significant shedding of L-selection, irrespective of applied artificial gravity. L-selectin is not affected by the overall immunity since no increased inflammatory parameters could be registered. A mechanical influence by fluid redistribution could be one possible explanation for the shedding, and further complimentary investigations are hence needed to define the role of CD62L not only as a marker of inflammatory reactions but possibly also as a marker for fluid shifts.

\section{ACKNOWLEDGMENTS}

The authors are grateful to the support from the European Space Agency (ESA) and to the Centre National d'Etudes Spatiales (CNES). The authors also highly acknowledge the professionalism and enthusiasm of the volunteers enrolled in the bed-rest study and are very grateful to the efficient and kind MEDES team. Special thanks have to be addressed to Gabi Gröger for technical assistance in measuring endothelial glycocalyx components.

\section{GRANTS}

This research was supported by the European Space Agency (ESA ELIPS 3 and 4 programs) and by the German National Space Program (DLR, 50WB0719/WB0919).

\section{DISCLOSURES}

No conflicts of interest, financial or otherwise, are declared by the author(s).

\section{AUTHOR CONTRIBUTIONS}

Author contributions: M.F. and A.C. conception and design of research; M.F., B.F., S.M., M.H., and A.C. performed experiments; M.F., B.F., S.M., M.H., and A.C. analyzed data; M.F., M.L., M.R., and A.C. interpreted results of experiments; M.F. and A.C. prepared figures; M.F., C.S., I.K., G.S., and A.C. drafted manuscript; M.F., B.F., M.L., C.S., I.K., G.S., M.R., and A.C. edited and revised manuscript; M.F., B.F., S.M., M.L., C.S., I.K., M.H., G.S., M.R., and A.C. approved final version of manuscript.

\section{REFERENCES}

1. Alves-Filho JC, de Freitas A, Spiller F, Souto FO, Cunha FQ. The role of neutrophils in severe sepsis. Shock 30, Suppl 1: 3-9, 2008.

2. Chappell D, Dorfler N, Jacob M, Rehm M, Welsch U, Conzen P, Becker BF. Glycocalyx protection reduces leukocyte adhesion after ischemia/ reperfusion. Shock 34: 133-139, 2010.

3. Chappell D, Jacob M, Hofmann-Kiefer K, Conzen P, Rehm M. A rational approach to perioperative fluid management. Anesthesiology 109: 723-740, 2008.

4. Chen H, Tian W, Lei S, Wei Y, Chen Y. [Effects of fluid shear stress on neutrophil surface expression of adhesion molecules]. J Biomed Eng 16: 288-294, 1999.

5. Chouker A, Thiel M, Baranov V, Meshkov D, Kotov A, Peter K, Messmer K, Christ F. Simulated microgravity, psychic stress, and immune cells in men: observations during 120-day 6 degrees HDT. $J$ Appl Physiol 90: 1736-1743, 2001.

6. Custaud MA, de Souza Neto EP, Abry P, Flandrin P, Millet C, Duvareille M, Fortrat JO, Gharib C. Orthostatic tolerance and spontaneous baroreflex sensitivity in men versus women after 7 days of headdown bed rest. Autonomic Neurosci 100: 66-76, 2002.

7. Dill DB, Costill DL. Calculation of percentage changes in volumes of blood, plasma, and red cells in dehydration. J Appl Physiol 37: 247-248, 1974.

8. Drummer C, Heer M, Joosten M, Stormer I, Hesse C, Beck L, Wolfram G, Baisch F. Regulation and distribution of body fluid during a 6-day head-down tilt study in a randomized cross-over design. J Gravit Physiol 7: 187-188, 2000.

9. Eltzschig HK, Carmeliet P. Hypoxia and inflammation. $N$ Engl $J$ Med 364: 656-665, 2011.

10. Feuerecker M, Mayer W, Kaufmann I, Gruber M, Muckenthaler F, Yi B, Salam AP, Briegel J, Schelling G, Thiel M, Chouker A. A corticoid-sensitive cytokine release assay for monitoring stress-mediated immune modulation. Clin Exp Immunol 172: 290-299, 2013.

11. Gmunder FK, Baisch F, Bechler B, Cogoli A, Cogoli M, Joller PW, Maass H, Muller J, Ziegler WH. Effect of head-down tilt bedrest (10 days) on lymphocyte reactivity. Acta Physiol Scand Suppl 604: 131-141, 1992.

12. Granger DN, Senchenkova E. Inflammation and the Microcirculation. San Rafael, CA: Morgan \& Claypool Life Sciences, 2010.

13. Jacob M, Bruegger D, Rehm M, Stoeckelhuber M, Welsch U, Conzen P, Becker BF. The endothelial glycocalyx affords compatibility of Starling's principle and high cardiac interstitial albumin levels. Cardiovasc Res 73: 575-586, 2007.

14. Jia XLC, J; Long M. IL-8-induced L-selectin shedding regulates its binding kinetics to PSGL-1. Chin Sci Bull 54: 8, 2009.

15. Johansen LB, Foldager N, Stadeager C, Kristensen MS, Bie P, Warberg J, Kamegai M, Norsk P. Plasma volume, fluid shifts, and renal responses in humans during $12 \mathrm{~h}$ of head-out water immersion. $J$ Appl Physiol 73: 539-544, 1992.

16. Johansen LB, Gharib C, Allevard AM, Sigaudo D, Christensen NJ, Drummer C, Norsk P. Haematocrit, plasma volume and noradrenaline in humans during simulated weightlessness for 42 days. Clin Physiol 17: 203-210, 1997.

17. Juliano RL. Signal transduction by cell adhesion receptors and the cytoskeleton: functions of integrins, cadherins, selectins, and immunoglobulin-superfamily members. Ann Rev Pharmacol Toxicol 42: 283-323, 2002.

18. Kaufmann I, Draenert R, Gruber M, Feuerecker M, Roider J, Chouker A. A new cytokine release assay: a simple approach to monitor the immune status of HIV-infected patients. Infection. In press. 
19. Kaufmann I, Hoelzl A, Schliephake F, Hummel T, Chouker A, Peter $\mathbf{K}$, Thiel M. Polymorphonuclear leukocyte dysfunction syndrome in patients with increasing sepsis severity. Shock 26: 254-261, 2006.

20. Kaufmann I, Schelling G, Eisner C, Richter HP, Beyer A, Krauseneck T, Chouker A, Thiel M. Decrease in adhesion molecules on polymorphonuclear leukocytes of patients with fibromyalgia. Rheumatol Int 29: 1109-1111, 2009.

21. Kelsen J, Bartels LE, Dige A, Hvas CL, Frings-Meuthen P, Boehme G, Thomsen MK, Fenger-Gron M, Dahlerup JF. 21 days head-down bed rest induces weakening of cell-mediated immunity: some spaceflight findings confirmed in a ground-based analog. Cytokine 59: 403-409, 2012.

22. Lee D, King MR. Shear-induced capping of L-selectin on the neutrophil surface during centrifugation. J Immunol Methods 328: 97-105, 2007.

23. Lee D, Schultz JB, Knauf PA, King MR. Mechanical shedding of L-selectin from the neutrophil surface during rolling on sialyl Lewis $X$ under flow. J Biol Chem 282: 4812-4820, 2007.

24. Martins PS, Kallas EG, Neto MC, Dalboni MA, Blecher S, Salomao R. Upregulation of reactive oxygen species generation and phagocytosis, and increased apoptosis in human neutrophils during severe sepsis and septic shock. Shock 20: 208-212, 2003.

25. Mitchell MJ, King MR. Shear-induced resistance to neutrophil activation via the formyl peptide receptor. Biophys J 102: 1804-1814, 2012.

26. Nandate K, Ogata M, Tamura H, Kawasaki T, Sata T, Shigematsu A. $\mathrm{N}$-acetyl-cysteine attenuates endotoxin-induced adhesion molecule expression in human whole blood. Anesthesia Analgesia 100: 1453-1457, 2005.

27. Paschall CD, Lawrence MB. L-selectin shear thresholding modulates leukocyte secondary capture. Ann Biomed Eng 36: 622-631, 2008.

28. Pavy-Le Traon A, Heer M, Narici MV, Rittweger J, Vernikos J. From space to Earth: advances in human physiology from 20 years of bed rest studies (1986-2006). Eur J Appl Physiol 101: 143-194, 2007.

29. Pfafflin A, Schleicher E. Inflammation markers in point-of-care testing (POCT). Analytic Bioanalytic Chem 393: 1473-1480, 2009.
30. Pries AR, Secomb TW, Gaehtgens P. The endothelial surface layer Pflügers Arch 440: 653-666, 2000.

31. Schmitt DA, Schwarzenberg M, Tkaczuk J, Hebrard S, Brandenberger G, Mauco G, Cogoli-Greuter M, Abbal M. Head-down tilt bed rest and immune responses. Pfiugers Arch 441: 79-84, 2000.

32. Shearer WT, Zhang S, Reuben JM, Lee BN, Butel JS. Effects of radiation and latent virus on immune responses in a space flight model. $J$ Allergy Clin Immunol 115: 1297-1303, 2005.

33. Sigaudo D, Fortrat JO, Maillet A, Allevard AM, Pavy-Le Traon A, Hughson RL, Guell A, Gharib C, Gauquelin G. Comparison of a 4-day confinement and head-down tilt on endocrine response and cardiovascular variability in humans. Eur J Appl Physiol Occup Physiol 73: 28-37, 1996.

34. Simon SI, Burns AR, Taylor AD, Gopalan PK, Lynam EB, Sklar LA, Smith CW. L-selectin (CD62L) cross-linking signals neutrophil adhesive functions via the Mac-1 (CD11b/CD18) beta 2-integrin. J Immunol 155: 1502-1514, 1995.

35. Sonnenfeld G, Butel JS, Shearer WT. Effects of the space flight environment on the immune system. Rev Environ Health 18: 1-17, 2003.

36. Springer TA. Traffic signals on endothelium for lymphocyte recirculation and leukocyte emigration. Ann Rev Physiol 57: 827-872, 1995.

37. Tarbell JM. Shear stress and the endothelial transport barrier. Cardiovasc Res 87: 320-330, 2010.

38. Uchakin PN, Cubbage ML, Sams CF, Morukov BV, Larina IV, Bobrovnik EB. Effects of the 120 days of head-down bed rest on cytokine secretion and its in vitro modulation by glucocorticoids. J Grav Physiol 5: P171-P172, 1998.

39. Weinbaum S, Tarbell JM, Damiano ER. The structure and function of the endothelial glycocalyx layer. Ann Rev Biomed Eng 9: 121-167, 2007.

40. Yang CB, Wang YC, Gao Y, Geng J, Wu YH, Zhang Y, Shi F, Sun XQ. Artificial gravity with ergometric exercise preserves the cardiac, but not cerebrovascular, functions during 4 days of head-down bed rest. Cytokine 56: 648-655, 2011.

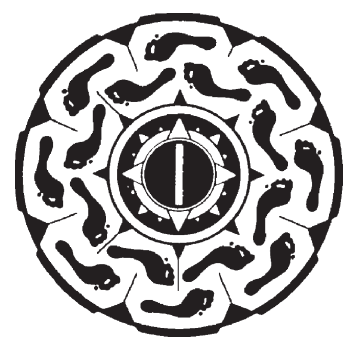

